Departamento de Ciencias Ambientales, Facultad de Recursos Naturales, Universidad Católica de Temuco. Temuco, Chile.

${ }^{2}$ Investigador independiente. ${ }^{a}$ Geógrafo.

${ }^{b}$ Magíster en Planificación y Gestión Territorial.

¿Licenciado en Ciencia Política, Magíster en Gobierno y Sociedad. Doctorante Ciencias Sociales, Universidad de la Frontera. Temuco, Chile.

Trabajo no recibió financiamiento. Los autores declaran no tener conflictos de interés.

Recibido el 2 de julio de 2020, aceptado el 28 de abril de 2021 .

Correspondencia a: Nelson Leonardo Sánchez Alarcón Rudecindo Ortega 2950, Temuco, Araucanía, Chile. nelsonsanchez.al@gmail.com

\section{El suicidio en la Región de La Araucanía: dinámica socioespacial durante los años 2004-2015}

\author{
NELSON SÁNCHEZ-ALARCÓN ${ }^{1, a}$, JUAN PABLO FRICK RAGGI ${ }^{1, a, b}$, \\ FRANCISCO VEGA DUARTE2,c
}

\section{Suicide rates in the Araucania Region of Chile}

Background: Suicide is a public health problem in the world. In Chile, suicide rates increased since 1990 with regional differences in rates. Aim: To analyze the geographical distribution of deaths due to suicide that occurred in the Region of Araucanía between the years 2004-2015. Material and Methods: The geographic distribution of suicide in the Araucania Region at district level (2004-2015) was analyzed using death data available at the web page of the Ministry of Health. Socio-demographic variables of suicide were characterized. The spatial distribution of suicide rates was represented using cartography, and suicide spatial clusters were identified through spatial-temporal exploration. Results: There were 1,562 suicides, $86 \%$ in men, with a mean rate of 13.07 $x 105$ inhabitants. The highest rates were registered in people aged over 70 years. Most individuals committing suicide were active workers. Hanging was the most common method to accomplish suicide. The territories from the coastal area in Cautin, and Nahuelbuta registered the highest suicide rates. Two statistically significant conglomerates with high incidence of suicide cases were identified. Conclusions: The use of geographic methods and the disaggregation of suicide cases at district level, allowed the identification of territorial variability in the distribution of suicide rates within the Araucanía Region. Spatial patterns of areas with high suicide risk were found.

(Rev Med Chile 2021; 149: 1004-1013)

Key words: Chile; Epidemiological Factors; Mortality; Suicide.

\section{L} a palabra suicidio, etimológicamente, es resultante de la unión de las palabras latinas sui (sí mismo) y cidium que es el acto de matar, que, a su vez, proviene de caedere (matar), literalmente "acción de matarse a sí

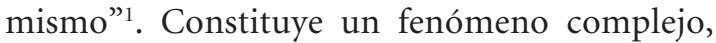
entendiéndose como "el acto humano consciente de causar la cesación de la propia vida"2, causado por un malestar pluridimensional que conduce a un individuo a un elevado deseo de morir ${ }^{2,3}$. Muchos suicidios se producen en momentos de crisis que deterioran la capacidad de una persona para afrontar las tensiones de la vida ${ }^{4}$ y en donde múltiples factores de riesgo individuales y sociales interactúan, tales como características demográficas, pobreza, enfermedades psiquiátricas, intentos suicidas previos, factores genéticos, eventos vitales negativos, desempleo, entre otros ${ }^{5-7}$.

Actualmente, más de 800.000 personas mueren al año por suicidio, siendo reconocido por la Organización Mundial de la Salud (OMS) como un problema de salud pública en el mundo; aproximadamente, cada 40 segundos se suicida alguien en el mundo ${ }^{6}$. En el año 2016 se registró una tasa mundial de 10,6 suicidios por cada 100.000 habitantes, siendo la segunda causa de muerte a nivel mundial en el rango de edades de 15-29 años ${ }^{4,8}$.

En Chile se registró un marcado incremento en 
las tasas de suicidio a partir de 1990; aumentando desde 5,6 por $100.000 \mathrm{~h}$ hasta alcanzar un máximo de 12,9 por $100.000 \mathrm{~h}$ en el año 2008, para luego disminuir levemente y estabilizarse durante los años posteriores, registrándose una tasa de 10,2 por $100.000 \mathrm{~h}$ en el año 2016, similar a la registrada a nivel mundial9,10. En Sudamérica, Chile se ha mantenido desde el año 2000 como el tercer país con las tasas más altas de suicidio, solo siendo superado por Bolivia y Uruguay ${ }^{8}$. A nivel interno, existe variabilidad en las tasas de suicidio por regiones, registrándose tasas más altas en la zona sur, siendo Los Lagos $\left(14,6 \times 10^{5} \mathrm{~h}\right)$ y La Araucanía $\left(12,9 \times 10^{5}\right.$ h) las regiones con las tasas más altas de suicidio en el país durante los años 2011-2017 $7^{10,11}$.

La prevalencia y características del comportamiento suicida varían entre diferentes áreas geográficas y grupos demográficos, posiblemente debido a diferencias socioeconómicas, culturales y de acceso a atención especializada ${ }^{6,12}$. En relación a esto, visualizar la distribución espacial de casos, identificando áreas de alto riesgo y patrones espaciales del suicidio, puede aportar a la toma de decisiones y planificación en salud pública, permitiendo analizar dónde están ocurriendo los eventos, cuáles son las poblaciones afectadas y qué tan cercanas están, para así proporcionar información de apoyo en programas de prevención, vigilancia y control del suicidio ${ }^{13-18}$.

El estudio del suicidio utilizando métodos geográficos registra solo un antecedente previo en la Región de La Araucanía, con una investigación que visualizó en mapas las tasas de suicidio por comuna para 3 años distintos (1992, 2005, 2009), observándose diferentes distribuciones para cada año, sin un patrón espacial definido ${ }^{19}$. Más allá, no hay antecedentes en Chile de investigaciones que abarquen la dimensión espacial del suicidio a través del uso de técnicas de análisis espacial. Es así como este estudio se propuso como objetivo principal analizar la distribución geográfica por comuna de las muertes por suicidio ocurridas en la Región de La Araucanía durante el período 2004-2015, además de caracterizar y describir las tendencias del suicidio a nivel regional.

\section{Material y Método}

Se realizó un estudio descriptivo exploratorio, basado en un diseño cuantitativo no experimental.
Los sujetos correspondieron a todos los casos de suicidio ocurridos durante los años 2004-2015 en la Región de la Araucanía. Se seleccionaron los casos de defunciones clasificadas como "lesiones autoinflingidas intencionalmente", registrados bajo los códigos de causa de muerte X60-X84 de la CIE- $10^{20}$ y obtenidos a partir de bases de datos de defunciones anuales proporcionada por el Departamento de Estadísticas e Informaciones de Salud (DEIS) del Ministerio de Salud.

Para cada caso, se obtuvieron variables generales (año, sexo, edad) y sociodemográficas (estado civil, nivel educacional, ocupación, área de residencia, método suicida). La edad se clasificó en rangos, según Martín Ruiz ${ }^{21}$.

Para caracterizar y describir las tendencias, se realizó un análisis descriptivo de todas las variables, presentándose tasas, frecuencias y porcentajes. Se calcularon tasas de mortalidad por suicidio generales en nivel de agregación regional y comunal, y tasas de mortalidad por suicidio específicas para las variables sexo y edad, expresadas cada 100.000 habitantes. Las tasas se estandarizaron en base al método directo con la población estándar mundial de la OMS (años 2000-2025) 22 . La caracterización de los datos fue realizada con el software Microsoft Excel y representados a través de tablas y gráficos. El ajuste de tasas se realizó con el software Epidat 4.2.

Se analizó el suicidio desde una perspectiva espacial a través de la utilización de la técnica "exploración espacio-temporal" para determinar la existencia de agrupaciones (conglomerados) espaciales estadísticamente significativas de alto riesgo de suicidio mediante la utilización del software SaTScan ${ }^{\mathrm{TM}} 9.6^{23}$. El análisis se realiza a través de la creación de ventanas circulares de exploración de distinto tamaño y ubicación, abarcando distintos conjuntos de ubicaciones y datos dentro de ellas, siendo cada ventana un posible conglomerado. El programa realiza una comparación (función de verosimilitud) entre el número de casos observado y el esperado en cada ventana según la distribución de Poisson, buscando el grupo en el que es menos probable que la distribución de casos haya ocurrido por casualidad, rechazando la hipótesis nula que indica que el número de casos esperado en cada ventana es proporcional a su tamaño poblacional (riesgo constante). Esto permite identificar agrupaciones de casos estadísticamente significativas con un valor $\mathrm{p}$ 0,05, asignado por 
la prueba Montecarlo (9999 simulaciones) ${ }^{23}$. Se realizó una exploración espacial para el período 2004-2015, fijándose como límite máximo para las ventanas de exploración un tamaño que no abarque más de $50 \%$ de la población total regional (población en riesgo).

Las tasas estandarizadas por comuna para el período señalado y las agrupaciones espaciales estadísticamente significativas fueron espacializadas en cartografías utilizando el software ArcGis ${ }^{\circledR}$ 10.4.

\section{Resultados}

Durante el período 2004-2015, se registraron 1.562 muertes por suicidio en La Araucanía. El $86,3 \%$ de los suicidios ( 1.348 casos) correspondió a hombres y $13,17 \%$ (214 casos) a mujeres. La media de edad general de los casos fue de 43,72 años; en hombres de 44,4 años y en mujeres de 39,1 años. La tasa ajustada de mortalidad por suicidio promedio para los años 2004-2015 fue de 13,07 por $100.000 \mathrm{~h}$. Se observó una tendencia estable en las tasas hasta el año 2011, para luego caer y volver a aumentar en los últimos dos años del período (Figura 1).

Según rangos de edad, el promedio de tasas para el período estudiado aumentó con la edad (Figura 2); las tasas más altas correspondieron a los rangos de 70-79 y 80 o más años. En hombres, la tendencia fue similar, mientras que en mujeres las tasas más altas se observaron en los rangos de 15-20 años (adolescencia) y 50-59 años. Se registró una tendencia temporal de aumento en las tasas de los rangos de mayor edad, duplicándose entre el año 2004 y 2015, observándose un aumento en las tasas desde 16,5 a 38,1 por $100.000 \mathrm{~h}$ en el rango 70-79 años y desde 12,5 a 29,6 por 100.000 $\mathrm{h}$ en el rango de 80 o más años. En adolescentes se observó una estabilización de las tasas a partir del año 2007, y una disminución en los últimos 2 años del período (Figura 3).

Al caracterizarse el suicidio en La Araucanía, se observa, según estado civil, una mayor proporción de suicidios cometidos por hombres solteros $(53,2 \%)$ versus casados $(42,8 \%)$, mientras que en las mujeres, la proporción fue levemente mayor en las casadas $(47,9 \%)$ respecto a solteras $(46,3 \%)$. En relación al nivel educacional, la mayor proporción de casos en hombres registraron como máximo nivel educacional el "básico o primario" $(53,1 \%)$, mientras que en mujeres predominó el nivel "medio" $(38,8 \%)$. La proporción de casos cuyo nivel educacional registrado fue "universitario" fue notablemente más alto en mujeres que en hombres (Tabla 1).

De acuerdo a la actividad desempeñada, existe una alta proporción de casos "laboralmente activos" (60,7\%); al desagregar por sexo, existen notables diferencias en las proporciones, en el caso

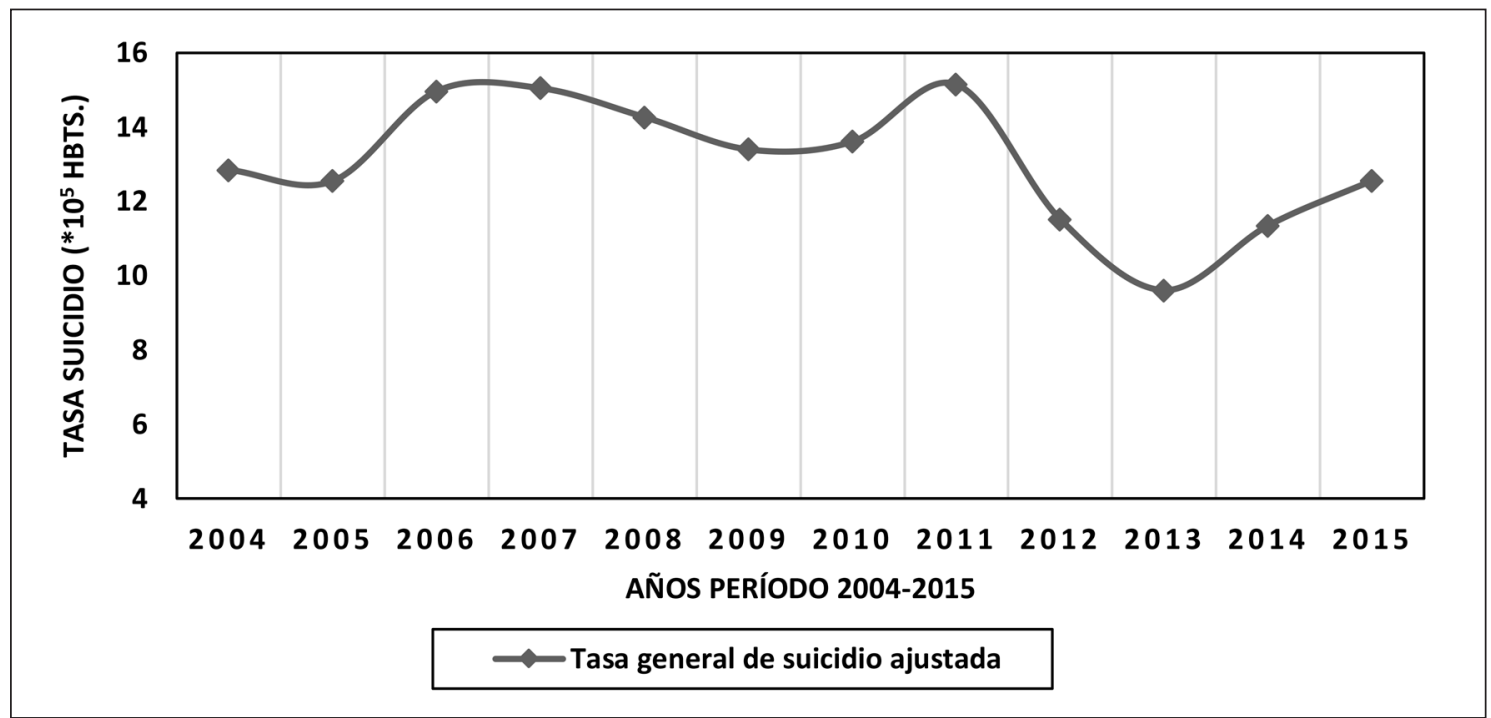

Figura 1. Tasa general de mortalidad por suicidio (x105 hbts) en la Región de La Araucanía (2004-2015). Datos de MINSAL e INE. 


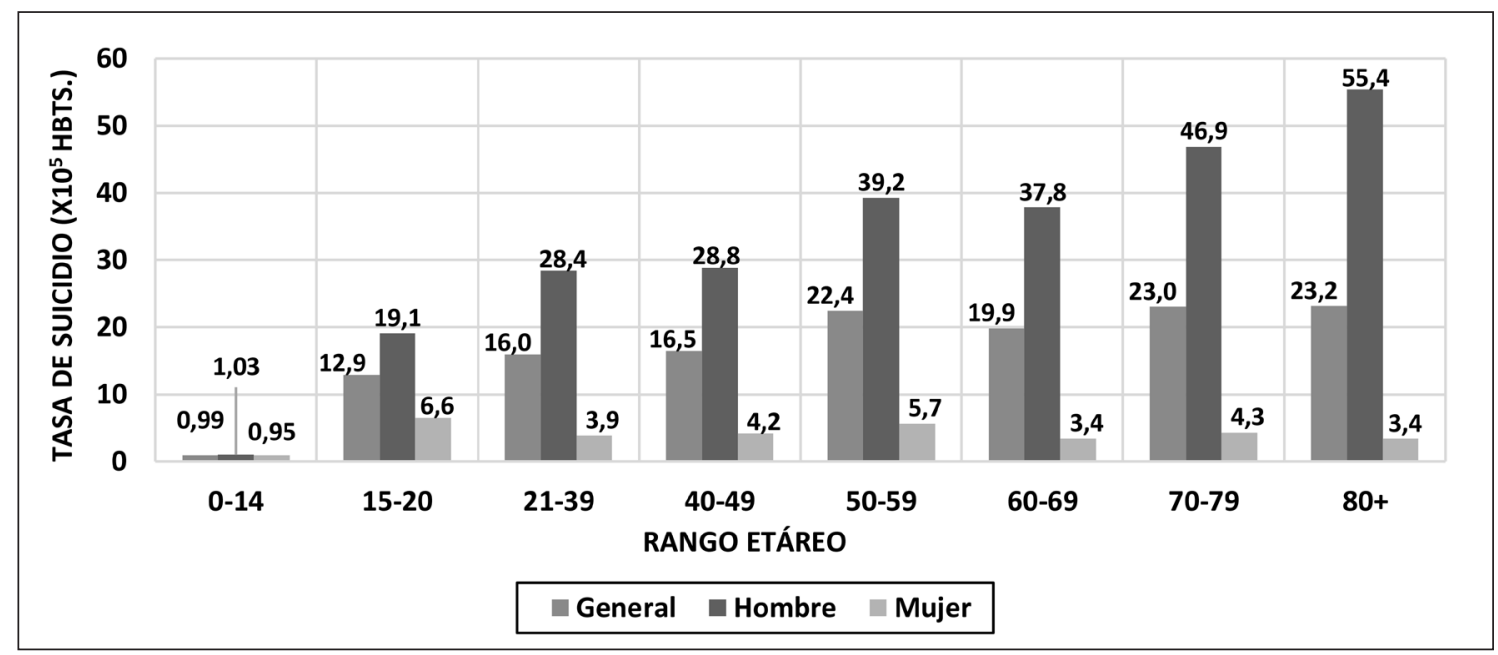

Figura 2. Distribución de tasas de suicidio promedio según rango de edad y sexo en la Región de La Araucanía (2004-2015). Datos de MINSAL e INE.

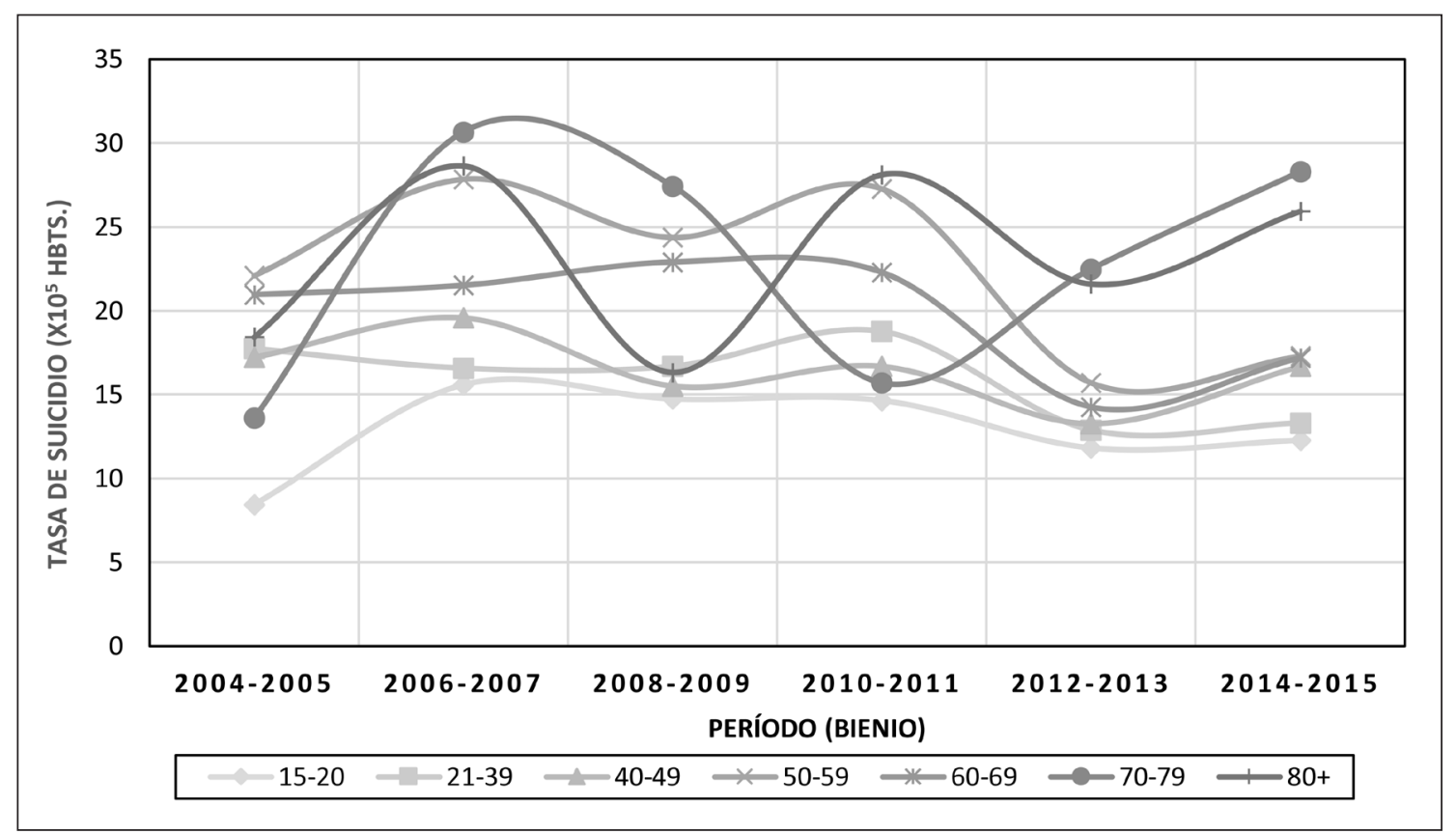

Figura 3. Evolución (bienios) de las tasas de suicidio según rango de edad en la Región de La Araucanía (2004-2015). Datos de MINSAL e INE.

de hombres un gran porcentaje de individuos se encontraba laboralmente activos $(67,1 \%)$, mientras que la gran mayoría de mujeres se encontraba inactivas (79\%), representadas en su mayoría por quienes desempeñaban labores de casa (43,9\%). El 5,8\% del total de casos correspondió a "cesantes", de los cuales solo se registró 1 mujer. Ocupaciones asociadas a niveles educacionales más altos (profesionales, técnicos medios) presentaron los menores porcentajes de suicidios. Un porcentaje importante de casos $(18,6 \%)$ correspondió a "jubilados" (Tabla 1). 
Tabla 1. Caracterización según variables, de los suicidios ocurridos en la Región de La Araucanía (2004-2015). Datos de Ministerio de Salud

\begin{tabular}{|c|c|c|c|c|c|c|c|}
\hline \multirow[t]{2}{*}{ Variable } & & \multicolumn{2}{|c|}{ Hombres } & \multicolumn{2}{|c|}{ Mujeres } & \multicolumn{2}{|c|}{ Total } \\
\hline & & $\mathbf{n}$ & $\%$ & $\mathbf{n}$ & $\%$ & $\mathbf{n}$ & $\%$ \\
\hline \multirow[t]{2}{*}{ Área de residencia } & Urbano & 749 & 55,6 & 147 & 68,7 & 896 & 57,4 \\
\hline & Rural & 599 & 44,4 & 67 & 31,3 & 666 & 42,6 \\
\hline \multirow[t]{5}{*}{ Estado civil } & Soltero & 707 & 54,2 & 87 & 46,3 & 794 & 53,2 \\
\hline & Casado & 559 & 42,8 & 90 & 47,9 & 649 & 43,5 \\
\hline & Ignorado & 8 & 0,6 & 5 & 2,7 & 31 & 2,1 \\
\hline & Viudo & 26 & 2 & 5 & 2,7 & 13 & 0,9 \\
\hline & Divorciado & 5 & 0,4 & 1 & 0,5 & 6 & 0,4 \\
\hline \multirow[t]{6}{*}{ Nivel educacional } & Básico o primario & 716 & 53,1 & 80 & 37,4 & 796 & 51 \\
\hline & Medio & 411 & 30,5 & 83 & 38,8 & 494 & 31,6 \\
\hline & Secundaria & 64 & 4,7 & 4 & 1,9 & 68 & 4,4 \\
\hline & Superior & 78 & 5,8 & 35 & 16,4 & 68 & 7,2 \\
\hline & Ninguno & 78 & 5,8 & 12 & 5,6 & 90 & 5,8 \\
\hline & Ignorado & 1 & 0,1 & --- & --- & 796 & 0,1 \\
\hline \multirow[t]{3}{*}{ Actividad } & Activo & 904 & 67,1 & 44 & 20,6 & 948 & 60,7 \\
\hline & Cesante o desocupado & 89 & 6,6 & 1 & 0,5 & 90 & 5,8 \\
\hline & Inactivo & 355 & 26,3 & 169 & 79 & 524 & 33,5 \\
\hline \multirow[t]{10}{*}{ Método } & Ahogamiento y sumersión & 12 & 0,9 & 6 & 2,8 & 18 & 1,2 \\
\hline & Ahorcamiento & 1.167 & 86,6 & 172 & 80,4 & 1.339 & 85,7 \\
\hline & Colisión intencional vehículo & --- & --- & 1 & 0,5 & 1 & 0,1 \\
\hline & Disparo de armas de fuego & 95 & 7 & 6 & 2,8 & 101 & 6,5 \\
\hline & Envenenamiento & 37 & 2,7 & 21 & 9,8 & 58 & 3,7 \\
\hline & No especificado & 10 & 0,7 & 3 & 1,4 & 13 & 0,8 \\
\hline & Objeto cortante & 12 & 0,9 & 2 & 0,9 & 14 & 0,9 \\
\hline & Por humo, fuego y llamas & 6 & 0,4 & --- & --- & 6 & 0,4 \\
\hline & Por material explosivo & 1 & 0,1 & --- & --- & 1 & 0,1 \\
\hline & Saltar de un lugar elevado & 8 & 0,6 & 3 & 1,4 & 11 & 0,7 \\
\hline
\end{tabular}

El método suicida más utilizado por amplia mayoría en ambos sexos fue el ahorcamiento, seguido en hombres por el disparo de armas de fuego $(7 \%)$ y en mujeres por envenenamiento $(9,8 \%)$ (Tabla 1$)$.

Las tasas por 100.00 habitantes ajustadas de suicidio (clasificadas en rangos según cuartiles) a nivel comunal para el período estudiado (Figura 4) variaron entre 6,3 (Melipeuco) y 24,93 (Teodoro Schmidt). Se observó que las tasas del cuartil superior se concentraron en comunas de la zona costera (Saavedra, Carahue, Teodoro Schmidt), el sector Nahuelbuta (Lumaco, Los Sauces, Purén) y la zona cordillerana (Lonquimay y Curarrehue). Las tasas más bajas se ubicaron principalmente en la zona de la depresión central, alrededor de Te- muco. Las comunas del cuartil superior destacaron por la alta proporción de suicidios ocurridos en áreas rurales, alcanzando en Lonquimay (89\%) y Saavedra (85\%) los mayores porcentajes.

El análisis espacial de conglomerados identificó tres agrupaciones con significancia estadística (p 0,05): dos de alto riesgo y una de bajo riesgo (Tabla 2). La agrupación principal de alto riesgo fue conformada por las comunas de Saavedra, Teodoro Schmidt y Carahue (RR: 1,86), y un conglomerado de alto riesgo secundario (RR: 1,34) fue conformado por las comunas de Angol, Renaico, Los Sauces, Purén, Ercilla, Traiguén, Lumaco, Collipulli, Victoria y Galvarino. La agrupación de bajo riesgo (RR: 0,61) fue conformada por las comunas de Temuco, Padre Las Casas y Cholchol (Figura 4). 


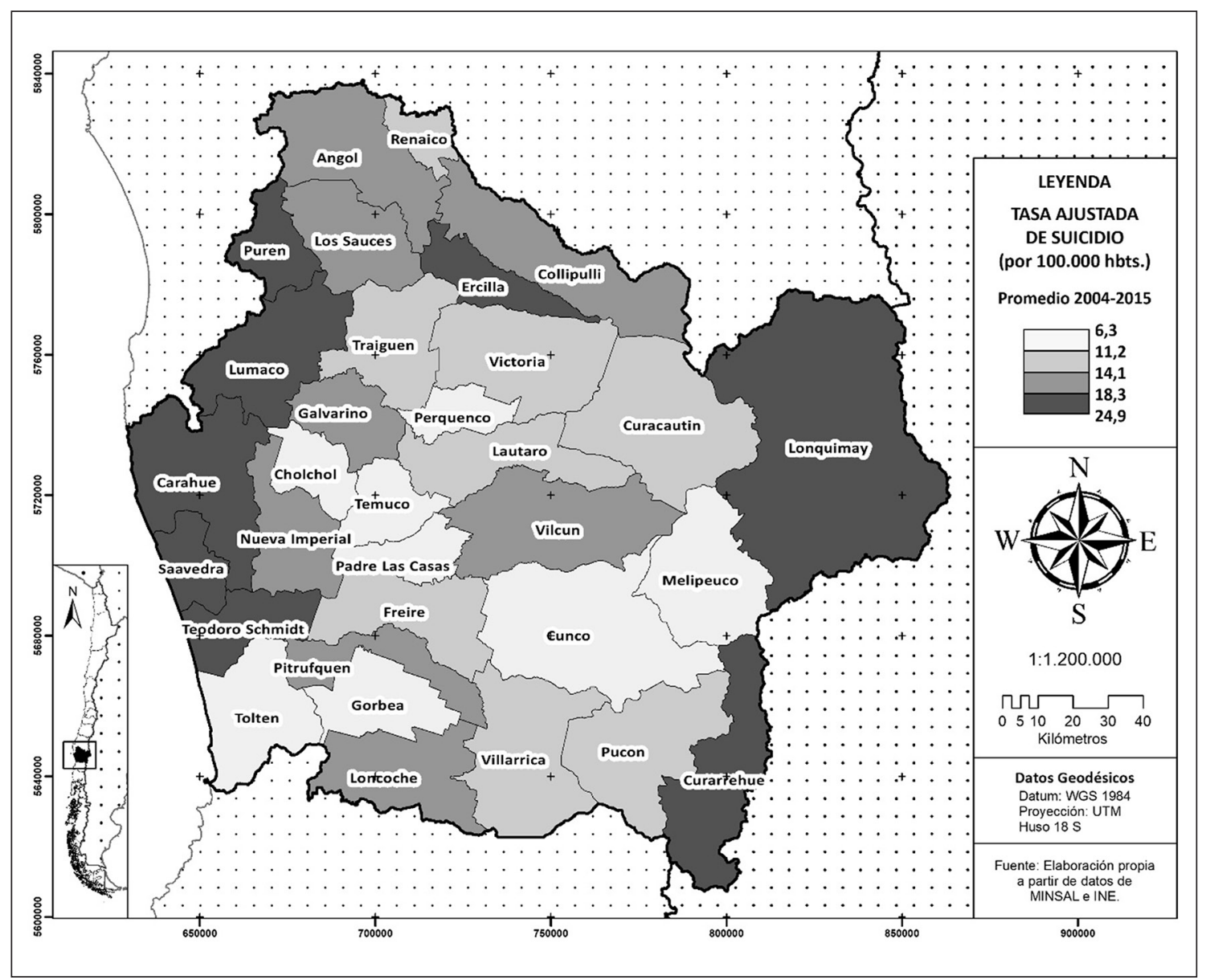

Figura 4. Mapa de las tasas de suicidio promedio en las comunas de la Región de La Araucanía (2004-2015), distribución según cuartiles. Datos de MINSAL e INE.

Tabla 2. Resultados de análisis SatScan de conglomerados de casos de suicidio en la Región de La Araucanía (2004-2015)

\begin{tabular}{|c|c|c|c|c|c|c|}
\hline Conglomerado & Comunas & $\begin{array}{l}\text { Casos } \\
\text { esperados }\end{array}$ & $\begin{array}{l}\text { Casos } \\
\text { ocurridos }\end{array}$ & $\mathbf{R R}^{*}$ & $\begin{array}{c}\text { Valor } \\
\text { p }\end{array}$ & $\begin{array}{c}\text { Tasa promedio } \\
\text { (x } 105 \mathrm{~h})\end{array}$ \\
\hline $\begin{array}{l}\text { Alto riesgo } \\
\text { (principal) }\end{array}$ & $\begin{array}{l}\text { Saavedra, Teodoro Schmidt, } \\
\text { Carahue }\end{array}$ & 92,57 & 164 & 1,86 & 0,0001 & 24,2 \\
\hline $\begin{array}{l}\text { Alto riesgo } \\
\text { (secundario) }\end{array}$ & $\begin{array}{l}\text { Angol, Renaico, Los Sauces, } \\
\text { Puren, Ercilla, Traiguén, Lumaco, } \\
\text { Collipulli, Victoria, Galvarino }\end{array}$ & 323,38 & 404 & 1,34 & 0,0003 & 17,0 \\
\hline Bajo riesgo & $\begin{array}{l}\text { Temuco, Padre Las Casas, } \\
\text { Cholchol }\end{array}$ & 590,89 & 425 & 0,61 & 0,0001 & 9,8 \\
\hline
\end{tabular}

\footnotetext{
*RR: Riesgo relativo.
} 


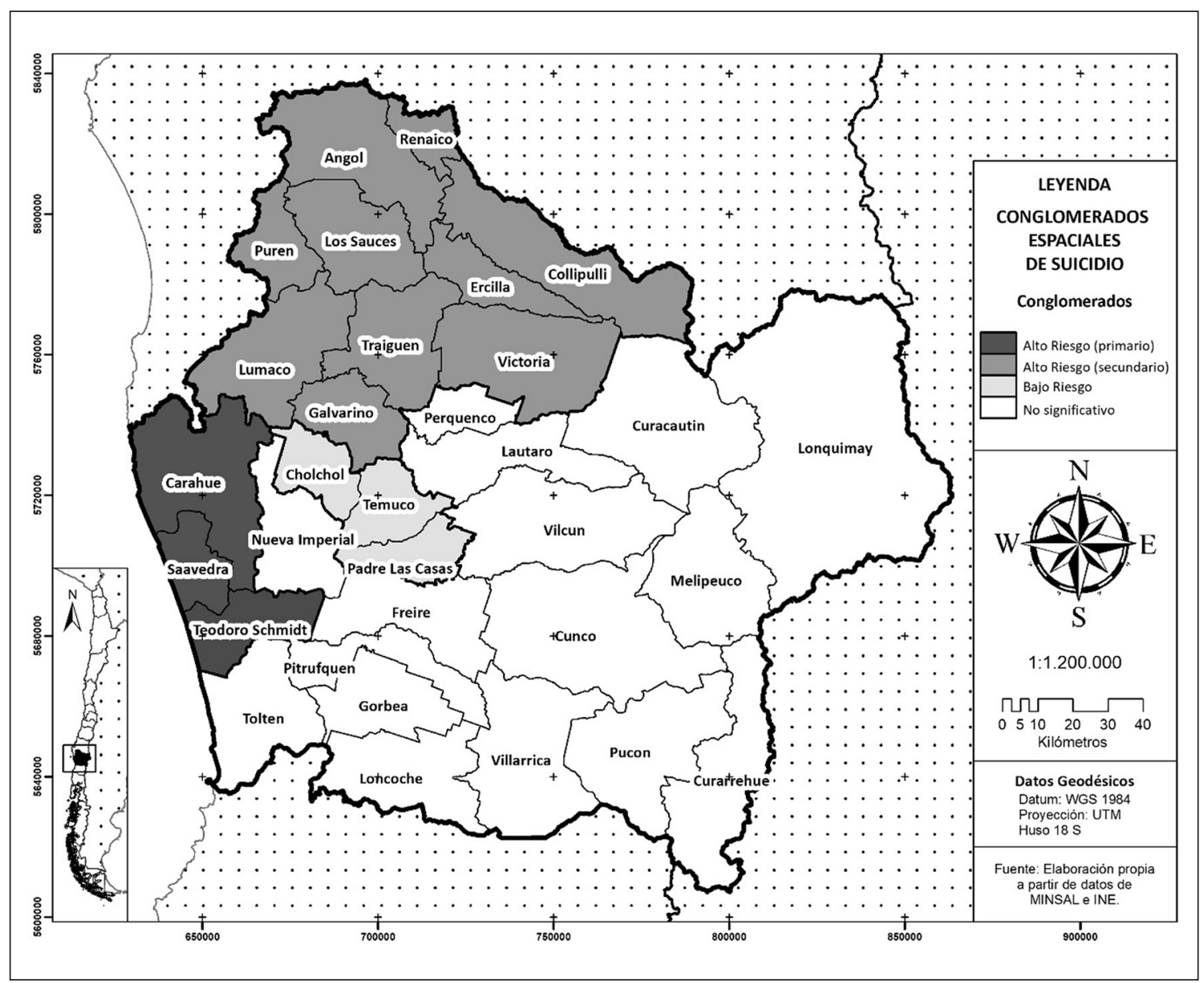

Figura 5. Mapa de ubicación de los conglomerados espaciales de suicidio en la Región de La Araucanía (2004-2015).

\section{Discusión}

La Región de La Araucanía presentó tasas de suicidio más altas que las registradas en promedio a nivel nacional para el período $2004-2015^{11}$, mientras que la tasa de suicidio en el año 2015 fue superior a la registrada en las Américas ${ }^{8}$. El patrón de suicidio, notablemente más numerosos en hombres, coincide con las tendencias registradas en Chile $e^{9,10,11}$ y a nivel mundial ${ }^{6,24,25,26}$. China es el único país en donde la tasa de suicidio es más alta en mujeres ${ }^{27}$.

Las tendencias de edad desagregadas según sexo son similares a las observadas a nivel nacional en hombres y mujeres ${ }^{7,24}$. Asimismo, el aumento observado en las tasas de suicidio en relación a la edad en La Araucanía es similar con la tendencia nacional ${ }^{7}$, lo que se ve reflejado en el importante porcentaje de casos de suicidio de personas jubiladas. Es preocupante la tendencia de aumento en las tasas registrada en mayores de 70 años, por lo que se hace imperativo profundizar en las características y factores de riesgo del suicidio en adultos mayores.

Una limitación de los estudios epidemiológicos sobre el suicidio es la dificultad para el registro de casos, ya que es un procedimiento complejo que abarca distintos niveles y criterios $^{6}$, existiendo circunstancias tales como la identificación médica de la causa de muerte, que pueden dificultar el diagnóstico ${ }^{7}$ y posterior registro de los casos, pudiendo subestimarse las cifras, especialmente en niños y adolescentes ${ }^{28}$. 
La estructura de la proporción de suicidios según actividad laboral es similar a la tendencia observada a nivel nacional ${ }^{9,24}$. Las características del trabajo, precarización e inseguridad laboral tienen consecuencias negativas en el bienestar y salud mental ${ }^{10,28}$; se sugiere indagar en las características de los vínculos y condiciones laborales (tipo, duración y estabilidad) y su influencia como posibles factores de riesgo suicida en la región. En relación a los suicidios cometidos por desempleados, la proporción es mayor en hombres, similar a la tendencia nacional ${ }^{7}$. Diversos autores señalan que el desempleo constituye un factor de riesgo suicida ${ }^{7,29,30}$, tomando relevancia la duración del desempleo y no solo el hecho en $\mathrm{si}^{1,31}$.

El ahorcamiento fue el método suicida más ampliamente utilizado por ambos sexos en La Araucanía, al igual que en las otras regiones del país y en las Américas $7,11,24,27,32$.

Los resultados del análisis espacial permitieron identificar diferencias en la distribución de las tasas de suicidio al interior de la Región de La Araucanía. En este sentido, el estudio de la distribución de fenómenos de salud en unidades territoriales con un menor nivel de agregación se realiza para explorar mejor las desigualdades entre distintos grupos de población, permitiendo visualizar diferencias territoriales que pueden permanecer invisibilizadas en niveles de agregación mayores ${ }^{33,34}$. La distribución espacial de la mortalidad por suicidio en un nivel de agregación comunal se caracterizó por su variabilidad, visualizándose áreas con tasas de suicidio más altas e identificándose agrupaciones de casos a través del análisis de conglomerados. Esto permite señalar diferencias en la incidencia del suicidio en la región, con la existencia de un patrón espacial de áreas de alto riesgo de suicidio que comprenden el área costera de la región (comunas de Carahue, Saavedra y Teodoro Schmidt) y, en menor medi$\mathrm{da}$, el sector de Nahuelbuta ubicado al noroeste (comunas de Angol, Renaico, Los Sauces, Purén, Ercilla, Traiguén, Lumaco, Collipulli, Victoria y Galvarino). Estas áreas registraron una mayor cantidad de casos de suicidio ocurridos en relación a los esperados para el período analizado.

Las áreas de alto riesgo corresponden a sectores con altos niveles de ruralidad y un bajo índice de desarrollo comunal ${ }^{35,36,37,38}$, por lo que sería relevante en futuras investigaciones profundizar en factores tales como los niveles de pobreza y condiciones materiales de vida, las estructuras productivas y relaciones laborales, la pertenencia de la población a pueblos indígenas, además de elementos estructurales como la conectividad, el gasto y equipamiento en salud o la dependencia de los habitantes a los fondos municipales. Estos elementos podrían expresar y contrastar ciertas características y diferencias territoriales que pondrían de manifiesto determinantes geográficas en los factores de riesgo suicida en la región y que podrían explicar los hallazgos de este estudio.

Debido al carácter metodológico de tipo descriptivo exploratorio, este estudio no buscó profundizar en factores y etiología del suicidio en el área de estudio, sino que pretende entregar datos para una aproximación inicial hacia la comprensión del fenómeno suicida en La Araucanía. Los resultados permiten visualizar la utilidad de los métodos geográficos en salud para identificar áreas de riesgo y potenciales inequidades en salud.

La naturaleza multifactorial del fenómeno suicida implica la necesidad de realizar un monitoreo permanente a las poblaciones en riesgo, incluyendo a quienes han incurrido en conducta suicida no letal, siendo importante tener un registro activo no solo de los suicidios consumados, sino también de los intentos suicidas, especialmente en las edades de riesgo identificadas. Los intentos suicidas son hasta 20 veces más frecuentes que los suicidios consumados ${ }^{39}$ y se dan con más frecuencia en mujeres, a pesar de que se concreta más en hombres ${ }^{24}$. Así, con información actualizada, detallada y especializada es posible contribuir a la toma de decisiones y formulación de políticas con pertinencia territorial enfocadas en la prevención del suicidio.

\section{Referencias}

1. Vega-Piñero M, Blasco-Fontecilla $\mathrm{H}$, Baca-García E, Díaz-Sastre C. El suicidio. Salud Global 2002; 4 (2): 1-15.

2. Muelas V, Mangado O. Consideraciones sobre el suicidio: Una perspectiva histórica. Psiquiatria.com 2007; 11 (3): $0-0$.

3. Baader T, Urra E, Millán R, Yáñez L. Algunas consideraciones sobre el intento de suicidio y su enfrentamiento. Rev Med Clin Condes 2011; 22 (3): 303-9.

4. OMS. Suicidio, datos y cifras. Organización mundial de 
la salud, 2019. Disponible en: https://www.who.int/es/ news-room/fact-sheets/detail/suicide [Consultado el 29 de mayo de 2020].

5. Gómez GA. Evaluación del riesgo de suicidio: Enfoque actualizado. Rev Med Clínica Las Condes 2012; 23 (5): 607-15.

6. OMS. Prevención del suicidio: un imperativo global. Washington DC; Organización Mundial de la Salud, 2014. Disponible en: https://apps.who.int/iris/bitstream/ handle/10665/136083/9789275318508_spa.pdf [Consultado el 16 de agosto de 2018].

7. Bogado M, Besa C, Rabat M, Reutter P, Carvajal C. Desempleo y Suicidio en Chile (1990-1991). Rev Gaceta de Psiquiatría Universitaria 2007; 3 (2): 156-62.

8. WHO. Global health observatory data repository. World Health Organization, 2017. Disponible en: https://apps. who.int/gho/data/view.main.MHSUICIDEREGv?lan$\mathrm{g}=$ en [Consultado el 29 de mayo de 2020].

9. Larraín A, Lobos F. Caracterización del suicidio en Chile: ¿qué nos dicen nuestras estadísticas? En: Guajardo G, Suicidios contemporáneos: vínculos, desigualdades y transformaciones socioculturales. Ensayos sobre Violencia, cultura y sentido. Santiago, Chile: Ediciones FLACSO-Chile 2017; 27-41.

10. Nahuelpán E, Varas J. El Suicidio en Chile: Análisis del Fenómenos desde los Datos Médicos Legales. Período 2000-2010. Actualización datos período 2011-2017. Disponible en: http://www.sml.gob.cl/dctos/genero/ INVESTIGACION_SUICIDIO\%20EN\%20CHILE\%20 2000-2010_ACTUALIZACION_version\%20final\%20. pdf [Consultado el 28 de mayo de 2020].

11. Otzen T, Sanhueza A, Manterola C, Escamilla-Cejudo JA. Mortalidad por suicidio en Chile: tendencias en los años 1998-2011. Rev Med Chile 2014; 142 (3): 305-13.

12. Alameda-Palacios J, Ruiz-Ramos M, García-Robredo B. Mortalidad por suicidio en Andalucía: distribución geográfica y relación con el uso de antidepresivos, la altitud y desigualdades socioeconómicas. Rev Esp Salud Pública 2015; 89 (3): 283-93.

13. Buzai G. Análisis espacial de la salud. En: Fuenzalida M, Buzai G, Moreno Jiménez A, García de León A. Geografía, geotecnología y análisis espacial: tendencias, métodos y aplicaciones. Santiago, Chile: Editorial Triángulo 2015; 188-207.

14. Ramírez ML. La moderna geografía de la salud y las tecnologías de la información geográfica. Rev Inv Ensayos Geográficos 2004; 4 (4): 53-64.

15. Loyola E, Castillo-Salgado C, Nájera-Aguilar P, Vidaurre M, Mujica OJ, Martínez-Piedra R. Los sistemas de información geográfica como herramienta para monitorear las desigualdades de salud. Rev Panam Salud Pública
2002; (12): 415-28.

16. Bailey TC. Spatial statistical methods in health. Cad de Saúde Pública 2001; 17 (5): 1083-98.

17. Valbuena-García AM, Rodríguez-Villamizar LA. Análisis espacial en epidemiología: revisión de métodos. Rev Univ Industrial de Santander 2018; 50 (4): 358-65.

18. Pina MF, Ferreira S, Correia AI, Castro A. Epidemiología espacial: nuevos enfoques para viejas preguntas. Universitas Odontológica 2010; 29 (63): 47-65.

19. Gaete A, Lara MR, Monsalves P. El suicidio en la Araucanía, años 1992-2009. Un enfoque interdisciplinario. En: Lara MR, Gaete A, Desviación social y delincuencia en Chile y la Araucanía. Temuco, Chile. Ediciones Universidad de la Frontera 2009; 245-79.

20. OPS. Clasificación Estadística Internacional de Enfermedades y Problemas Relacionados con la Salud, Décima Revisión (CIE-10). Organización Panamericana de la Salud, 2013. Disponible en: https://www. paho.org/hq/index.php?option=com_content \&view $=$ article\&id=9178:2013-actualizaciones-cie-10\&Itemid $=40350 \&$ lang $=$ es [Consultado el 14 de julio de 2018].

21. Martín JF. Los factores definitorios de los grandes grupos de edad de la población: tipos, subgrupos y umbrales. Rev Elec Scripta Nova 2005; 9 (190): 0-0.

22. WHO. World standard population. World Health Organization, 2014. Disponible en: http://apps.who.int/ healthinfo/statistics/mortality/whodpms/definitions/ pop.htm [Consultado el 4 de noviembre de 2018].

23. Kulldorf, M. SatScan ${ }^{\mathrm{TM}}$ User Guide for versión 9.6, 2018. Disponible en https://www.satscan.org/cgi-bin/satscan/ register.pl/SaTScan_Users_Guide.pdf?todo=process_ userguide_download [Consultado el 10 de agosto de 2018].

24. Baader T, Behne P, Molina JL, Gacitúa L, Yáñez L, Urra E, et al. ¿Está cambiando la prevalencia de los suicidios y sus características en la población chilena?: Análisis de las tasas de suicidios y sus características sociodemográficas, ocurridas en la provincia de Valdivia, actual Región de Los Ríos, entre los años 1996 a 2008. Rev Chil Neuro-Psiquiat 2011; 49 (3): 273-82.

25. Corona-Miranda B, Alfonso-Sagué K, Hernández-Sánchez M, Lomba-Acevedo P. Epidemiología del suicidio en Cuba, 1987-2014. MEDICC 2016; 18 (3): 15-20.

26. Hernández-Vásquez A, Azañedo D, Rubilar-González J, Huarez B, Grendas L. Evolución y diferencias regionales de la mortalidad por suicidios en el Perú, 20042013. Rev Peru Med Exp Salud Publica 2016; 33: 751-7.

27. Madariaga C, Ulloa O, Gómez AG, Iriondo P, Alvarado R. Muertes por suicidio en la Región de Tarapacá, años 1990-2013. Rev Chil Neuro-Psiquiat 2016; 54 (3): 250-8. 
28. Moyano E, Barria R. Suicidio y producto interno bruto (PIB) en Chile: Hacia un modelo predictivo. Rev Latinoam Psicol 2006; 38 (2): 343-59.

29. Qin P, Agerbo E, Mortensen P. Suicide risk in relation to socioeconomic, demographic, psychiatric and familial factors: A national register-nased study of all Suicides in Denmark 1981-1997. Am J Psychiatry 2003; 160 (4): 765-72.

30. Yoshimasu K, Kiyohara C, Miyashita K. Suicidal risk factors and completed suicide: meta-analyses based on psychological autopsy studies. Environ Health Prev Med. 2008; 13 (5): 243-56.

31. Classen TJ, Dunn RA. The effect of job loss and unemployment duration on suicide risk in the United States: A new look using mass-layoffs and unemployment duration. Health Economics 2012; 21 (3): 338-50.

32. OPS. Mortalidad por suicidio en las Américas, informe regional. Washington D.C.: Organización Panamericana de la Salud; 2014. Disponible en: http://www. bvsde.paho.org/documentosdigitales/bvsde/texcom/ PAHOMortalidad-suicidio.pdf [Consultado el 22 de agosto de 2018].

33. Metzger X. La agregación de datos en la medición de desigualdades e inequidades en la salud de las poblaciones. Rev Panam Salud Publica 2002; 12 (0): 445-53.

34. Sáenz Vela HM. Revisando los métodos de agregación de unidades espaciales: MAUP, algoritmos y un breve ejemplo. Estudios demográficos y urbanos 2016; 31 (2): 385-411.

35. Garín A, Moraga A, Salvo S. Pobreza y género: distribución espacial en la región de La Araucanía. Tiempo y espacio 2007; 17-19: 60-73.

36. Hernández J, Ramírez H, Parrao A, Salazar L, González J, Godoy C. Índice de desarrollo comunal, Chile 2020. Santiago (CH): Universidad Autónoma de Chile; 2020.

37. INE. Censo de población y vivienda 2017. Instituto Nacional de Estadísticas, 2017. Disponible en: https://www. censo2017.cl/ [Consultado el 29 de diciembre de 2020].

38. Garín C, Albers C, Ortega E. Las expresiones de la ruralidad en la región de La Araucanía, Chile, 1997-2007. Estudios Sociales 2011; 19: 67-89.

39. Echávarri O, Maino M, Fischman R, Morales S, Barros J. Aumento sostenido del suicidio en Chile: un tema pendiente. Centro de Políticas Públicas UC 2015; (79): 1-14. Disponible en https://politicaspublicas.uc.cl/ wp-content/uploads/2015/07/Nº-79. 